\title{
Communication
}

\section{Broadband Solutions of Potential Integral Equations With NSPWMLFMA}

\author{
Bahram Khalichi $^{\circledR}$, Özgür Ergül ${ }^{\circledR}$, and Vakur B. Ertürk
}

\begin{abstract}
In this communication, a mixed-form multilevel fast multipole algorithm (MLFMA) is combined with the recently introduced potential integral equations (PIEs), also called as the $A-\phi$ system, to obtain an efficient and accurate broadband solver that can be used for the solution of electromagnetic scattering from perfectly conducting surfaces over a wide frequency range including low frequencies. The mixed-form MLFMA uses the nondirective stable planewave MLFMA (NSPWMLFMA) at low frequencies and the conventional MLFMA at middle/high frequencies. Various numerical examples are presented to assess the validity, efficiency, and accuracy of the developed solver.
\end{abstract}

Index Terms-Fast solvers, low-frequency (LF) breakdown, nondirective stable plane-wave MLFMA (NSPWMLFMA), potential integral equations (PIEs).

\section{INTRODUCTION}

Frequency-domain surface integral equations (SIEs) used together with the method of moments (MoMs) [1], and/or its accelerated versions, such as the multilevel fast multipole algorithm (MLFMA) [2], [3], are usually the most promising choices in solving electromagnetic scattering problems including perfect electric conductors (PECs) or piecewise homogeneous penetrable objects. However, the electric-field integral equation (EFIE) (as one of the most popular SIEs) is susceptible to the well-known low-frequency (LF) breakdown problem, which prohibits its use at low frequencies and/or dense discretizations [4]. Although the magnetic-field integral equation (MFIE) is less affected from the LF breakdown, it is usually criticized for being less accurate [5], [6], which can be improved by using mixed discretizations [5], and being applicable only to closed surfaces. Several remedies to the LF breakdown problem of EFIE have been discussed in a detailed way in the literature [7]-[13]. The loop-star decomposition of basis functions is introduced for EFIE in [7], while the charge is used as an extra unknown and current continuity condition is employed in [8] and [9]. Furthermore, a Calderon preconditioner and a preconditioner based on a quasi-Helmholtz decomposition using hierarchical bases have been successfully employed in [10]-[13]. Recently, potential integral equations (PIEs) based on satisfying the boundary conditions (BCs) on the surface of the scatterer for the electric scalar potential $\phi$ and the magnetic vector potential $\boldsymbol{A}$ have been presented [14]-[16] for the solution of electromagnetic scattering problems at low frequencies. PIEs are shown to be immune to the LF breakdown problem without using hierarchical basis functions and/or a Calderon preconditioner. It has been demonstrated that accurate computation of the charge

Manuscript received August 1, 2018; revised January 11, 2019; accepted March 7, 2019. Date of publication March 18, 2019; date of current version May 31, 2019. This work was supported by the Scientific and Technical Research Council of Turkey (TÜBITAK) under Grant 117E113. (Corresponding author: Bahram Khalichi.)

B. Khalichi and V. B. Ertürk are with the Department of Electrical and Electronics Engineering, Bilkent University, Ankara 06800, Turkey (e-mail: bahram@ee.bilkent.edu.tr; vakur@ee.bilkent.edu.tr).

Ö. Ergül is with the Department of Electrical and Electronics Engineering, Middle East Technical University, Ankara 06800, Turkey (e-mail: ozergul@ metu.edu.tr)

Color versions of one or more of the figures in this communication are available online at http://ieeexplore.ieee.org.

Digital Object Identifier 10.1109/TAP.2019.2905965

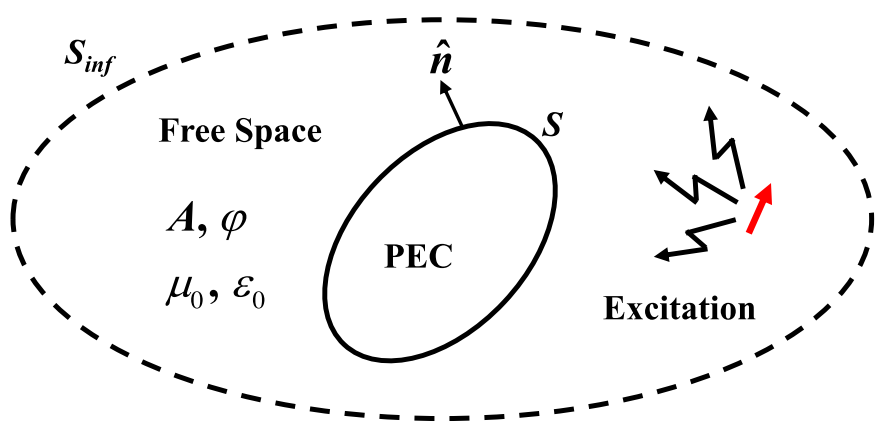

Fig. 1. Configuration of region and medium to obtain surface PIEs.

density, in addition to the current density, is possible by using PIEs at the cost of a solution of an additional integral equation [17].

In this communication, a mixed-form MLFMA is combined with the recently introduced PIEs, called as the $\boldsymbol{A}-\phi$ formulation [15], [16], to obtain an efficient and accurate broadband solver to solve electromagnetic scattering from multiscale PEC surfaces over a wide frequency range. The mixed-form MLFMA uses the conventional MLFMA at middle/high frequencies and the nondirective stable plane-wave MLFMA (NSPWMLFMA) [18]-[21] at low frequencies (i.e., electrically small boxes: box size $=B_{\lambda}<\lambda / 8$ ). NSPWMLFMA is based on shifting the integration path into the complex plane to include the evanescent waves for electrically short distances. Basically, in this algorithm, a closed-form expression for the translation operator along the $z$-axis is derived, and then stable translations in all other directions are achieved via a QR-decomposition-based method. Therefore, NSPWMLFMA is preferred in this study due to its error controllability and suitability for being combined with the conventional MLFMA to obtain a robust broadband mixed-form MLFMA solver for $\boldsymbol{A}-\phi$ formulations. Apart from NSPWMLFMA, there are many different studies available in the literature to treat the LF breakdown of MLFMA [22]-[29]. Among them, LF fast inhomogeneous plane-wave algorithm (LF-FIPWA) with diagonal translations can also be combined with the conventional MLFMA elegantly [23]-[25]. However, its directionality problem requires six different expansions of Green's function in six different directions to cover all translations in the entire space. Another interesting approach is interpolating MLFMA with a fast Fourier transform accelerator [29]. However, it may exhibit computational complexity issues for larger problems to maintain the interpolation accuracy [29].

The outline of this communication as follows. Section II presents the formulation of the problem containing PIEs, NSPWMLFMA, and a brief explanation of a preconditioning. The numerical results are provided in Section III, followed by our concluding remarks. An $e^{-i \omega t}$ time convention, where $\omega=2 \pi f$ with $f$ being the operating frequency, is assumed and suppressed throughout this communication.

\section{FORMULATION}

\section{A. Potential Integral Equations}

Fig. 1 illustrates a PEC scatterer $S$ with a unit normal vector $\hat{\boldsymbol{n}}$ in free-space $\left(\varepsilon_{0}\right.$ : permittivity, $\mu_{0}$ : permeability) illuminated by a 
source. Using the surface equivalence principle, generalized Green's theorem, and Lorentz gauge condition in conjunction with appropriate BCs for the magnetic vector potential $\boldsymbol{A}$ and the electric scalar potential $\phi$ on the surface of $S$ [15], PIEs in terms of $\boldsymbol{A}$ and $\phi$ can be derived as [15], [16]

$$
\begin{aligned}
0= & -\hat{\boldsymbol{n}} \times \hat{\boldsymbol{n}} \times \boldsymbol{A}_{\mathrm{inc}}(\boldsymbol{r})-\hat{\boldsymbol{n}} \times \hat{\boldsymbol{n}} \times \int_{S^{\prime}} d s^{\prime} \mu_{0} g\left(\boldsymbol{r}, \boldsymbol{r}^{\prime}\right) \boldsymbol{J}\left(\boldsymbol{r}^{\prime}\right) \\
& -\hat{\boldsymbol{n}} \times \hat{\boldsymbol{n}} \times \int_{S^{\prime}} d s^{\prime} \Sigma\left(\boldsymbol{r}^{\prime}\right) \nabla^{\prime} g\left(\boldsymbol{r}, \boldsymbol{r}^{\prime}\right) \\
0= & i \omega \mu_{0} \varepsilon_{0} \phi_{\mathrm{inc}}(\boldsymbol{r})+\int_{S^{\prime}} d s^{\prime} \mu_{0} g\left(\boldsymbol{r}, \boldsymbol{r}^{\prime}\right) \nabla^{\prime} \cdot \boldsymbol{J}\left(\boldsymbol{r}^{\prime}\right) \\
& +\omega^{2} \mu_{0} \varepsilon_{0} \int_{S^{\prime}} d s^{\prime} \Sigma\left(\boldsymbol{r}^{\prime}\right) g\left(\boldsymbol{r}, \boldsymbol{r}^{\prime}\right) .
\end{aligned}
$$

In (1), $\boldsymbol{A}_{\text {inc }}(\boldsymbol{r})$ and $\phi_{\text {inc }}(\boldsymbol{r})$ are the incident magnetic vector potential and the incident electric scalar potential, respectively. In addition, $g\left(\boldsymbol{r}, \boldsymbol{r}^{\prime}\right)$ is the free-space scalar Green's function given by

$$
g\left(\boldsymbol{r}, \boldsymbol{r}^{\prime}\right)=\frac{e^{i k\left|\boldsymbol{r}-\boldsymbol{r}^{\prime}\right|}}{4 \pi\left|\boldsymbol{r}-\boldsymbol{r}^{\prime}\right|}
$$

while $\boldsymbol{J}\left(\boldsymbol{r}^{\prime}\right)$ and $\Sigma\left(\boldsymbol{r}^{\prime}\right)=\hat{\boldsymbol{n}} \cdot \boldsymbol{A}\left(\boldsymbol{r}^{\prime}\right)$ are the unknowns to be found, namely, the surface electric current density and the normal component of the magnetic vector potential, respectively.

First, expanding $\boldsymbol{J}(\boldsymbol{r})$ with the Rao-Wilton-Glisson (RWG) basis functions [30] as

$$
\boldsymbol{J}(\boldsymbol{r})=\sum_{n=1}^{N} j_{n} \boldsymbol{b}_{n}(\boldsymbol{r})
$$

with $j_{n}$ being the unknown coefficients, and expanding $\Sigma(\boldsymbol{r})$ with pulse basis functions (on triangular supports $S_{m}$ ) as

$$
\Sigma(\boldsymbol{r})=\sum_{m=1}^{M} s_{m} \sigma_{m}(\boldsymbol{r}), \quad \sigma_{m}(\boldsymbol{r})= \begin{cases}1 / A_{m} & \boldsymbol{r} \in S_{m} \\ 0 & \boldsymbol{r} \notin S_{m}\end{cases}
$$

with $s_{m}$ being the unknown coefficients, and then testing (1a) with the RWG functions while (1b) with pulse functions, (1a) and (1b) can be cast into matrix form given by

$$
\left[\begin{array}{cc}
\overline{\boldsymbol{\Gamma}}_{\boldsymbol{J}, \boldsymbol{J}} & \overline{\boldsymbol{\Gamma}}_{\boldsymbol{J}, \sigma} \\
\overline{\boldsymbol{\Gamma}}_{\sigma, \boldsymbol{J}} & k_{0}^{2} \overline{\boldsymbol{\Gamma}}_{\sigma, \sigma}
\end{array}\right]\left[\begin{array}{l}
\boldsymbol{j} / c_{0} \\
\boldsymbol{S} / \eta_{0}
\end{array}\right]=-\left[\begin{array}{c}
\boldsymbol{a}_{\text {inc }} / \eta_{0} \\
i k_{0} \varepsilon_{0} \boldsymbol{\varphi}_{\text {inc }}
\end{array}\right] .
$$

In (5), $k_{0}, \eta_{0}$, and $c_{0}$ are the free-space wavenumber, intrinsic impedance, and wave velocity, respectively. The matrix entries of each submatrix in (5) are determined according to [15]. In (5), $\boldsymbol{j}=\left[j_{1}, j_{2}, \ldots, j_{N}\right]^{t}, \boldsymbol{s}=\left[s_{1}, s_{2}, \ldots, s_{M}\right]^{t}$ with $t$ denoting the transpose operator, and $\boldsymbol{a}_{\text {inc }}$ and $\boldsymbol{\varphi}_{\text {inc }}$ contain discrete tested form of $\boldsymbol{A}_{\text {inc }}(\boldsymbol{r})$ and $\phi_{\text {inc }}(\boldsymbol{r})$. Finally, $N$ and $M$ are the number of the RWGs and triangles, respectively.

\section{B. NSPWMLFMA for PIES}

The derivation of the conventional MLFMA implies that only the propagating part of the plane-wave spectrum is included for sufficient accuracy. This is simply achieved via use of the diagonalized form of Green's function given by

$$
\begin{aligned}
\frac{e^{i k R}}{4 \pi R} & =\frac{i k}{(4 \pi)^{2}} \int d^{2} \hat{k} e^{i k \hat{k} \cdot\left(\boldsymbol{v}_{1}+\boldsymbol{v}_{2}\right)} T(k \boldsymbol{w}, \theta, \varphi), \\
|\boldsymbol{w}| & >\left|\boldsymbol{v}_{\mathbf{1}}+\boldsymbol{v}_{\mathbf{2}}\right|
\end{aligned}
$$

where $R=\left|\boldsymbol{w}+\boldsymbol{v}_{\mathbf{1}}+\boldsymbol{v}_{\mathbf{2}}\right|, \quad d^{2} \hat{k}=\sin \theta d \theta d \varphi$, and $\hat{\boldsymbol{k}}=$ $\hat{\boldsymbol{a}}_{x} \sin \theta \cos \varphi+\hat{\boldsymbol{a}}_{y} \sin \theta \sin \varphi+\hat{\boldsymbol{a}}_{Z} \cos \theta$. In the above, $\boldsymbol{v}_{1}$ and $\boldsymbol{v}_{2}$ are the shift functions corresponding to aggregation and disaggregation stages, respectively, $\boldsymbol{w}$ is the translation vector, and $T(k \boldsymbol{w}, \theta, \varphi)$ is the translation operator defined as

$$
T(k \boldsymbol{w}, \theta, \varphi)=\sum_{l=0}^{\infty} i^{l}(2 l+1) h_{l}^{(1)}(k w) P_{l}(\hat{\boldsymbol{k}} \cdot \hat{\boldsymbol{w}})
$$

where $P_{l}(\hat{\boldsymbol{k}} \cdot \hat{\boldsymbol{w}})$ is the Legendre polynomial and $h_{l}^{(1)}(k w)$ is the spherical Hankel function of the first kind. Unfortunately, the conventional MLFMA exhibits LF breakdown as a result of numerical round-off errors that occur at lower frequencies or at the deeper levels in the corresponding tree structure [20]. In this scenario, $h_{l}^{(1)}(k w)$ increases exponentially for small translation distances (i.e., $|\boldsymbol{w}|=w)$ and cannot be compensated by exponential shift functions. This drawback of the conventional MLFMA is solved by using NSPWMLFMA [20] in this communication. Hence, NSPWMLFMA is combined with PIEs to form a mixed-form MLFMA for the first time to the best of our knowledge. Briefly, the $\theta$ direction of the integration over the Ewald sphere is changed form $[0, \pi]$ to $[0,2 \pi]$ as the first step. Then, the Fourier spectrum in $\theta$ is calculated analytically for the smooth translation along the $z$-direction and the integration path is deformed into the complex plane to include the evanescent waves for electrically short distances. Finally, translations along other directions are calculated using the rotation matrix $\overline{\mathbf{R}}_{v}$ leading to an expression for the free-space Green's function as

$$
\begin{gathered}
\frac{e^{i k R}}{4 \pi R}=\frac{i k}{(4 \pi)^{2}} \int_{0}^{2 \pi} \int_{0+i \chi}^{2 \pi+i \chi} d \theta d \varphi e^{i\left(\overline{\mathbf{R}}_{v} \cdot k \hat{\boldsymbol{k}}(\theta+i \chi, \varphi)\right) \cdot \boldsymbol{v}} \\
\cdot \underbrace{\sum_{n=-L}^{L} b_{n} e^{i n(\theta+i \chi)}}_{\tilde{T}_{Z}(k \boldsymbol{w}, \theta+i \chi, \varphi)=T_{Z}(k \boldsymbol{w}, \theta+i \chi, \varphi)|\sin (\theta+i \chi)|}
\end{gathered}
$$

In (8), $\boldsymbol{v}=\boldsymbol{v}_{1}+\boldsymbol{v}_{2}$ and $L$ is the truncation number that is a fixed value at low frequencies or at the deeper levels [31], [32]. In addition, $\chi$ indicates the magnitude of shifting into the complex plane and $b_{n}$ represents the Fourier coefficients. Optimal $\chi$ values and analytical expression of $b_{n}$ are provided in [20]. Making use of uniform integration for both $\theta$ and $\varphi$ directions, discretized form of (8) is obtained as

$$
\begin{gathered}
\frac{e^{i k R}}{4 \pi R} \simeq \frac{i k C}{(4 \pi)^{2}} \sum_{n_{\varphi}=1}^{N_{\varphi}} \sum_{n_{\theta}=1}^{N_{\theta}} e^{i\left(\overline{\mathbf{R}}_{v} \cdot k \hat{\boldsymbol{k}}_{n}\left(\theta_{n_{\theta}}+i \chi, \varphi_{n_{\varphi}}\right)\right) \cdot \boldsymbol{v}} \\
\cdot \sum_{n=-L}^{L} b_{n} e^{i n\left(\theta_{n_{\theta}}+i \chi\right)} \quad \forall n_{\theta} \in\left[1, N_{\theta}\right], \forall n_{\varphi} \in\left[1, N_{\varphi}\right] \\
N_{\theta}=N_{\varphi}=(2 L+2)
\end{gathered}
$$

where $C$ is a constant, and $N_{\theta}$ and $N_{\varphi}$ indicate the number of discretization points for $\theta$ and $\varphi$ directions, respectively. At this point, use of (8) [or its discretized form (9)] for all translations is inefficient as it requires the rotation of $k$-space and all vectors for each translation (which is equivalent to the rotation of the entire integration points during the numerical integration). Moreover, aggregation and disaggregation at deeper levels are not possible since it requires re-aggregation and re-disaggregation of basis and testing patterns, respectively, for each specific translation. These problems are solved by expanding the shift functions in (8) in terms of spherical harmonics and applying QR-decomposition algorithm, which permits one to define newly discretized $k$-vectors (as a span of entire $k$-space) for stable translations in all other directions without rotating basis and testing vectors and $k$-space [20]. Consequently, for $p$ distinct sources, $J_{p}$, to be aggregated, translated, and disaggreagated to 


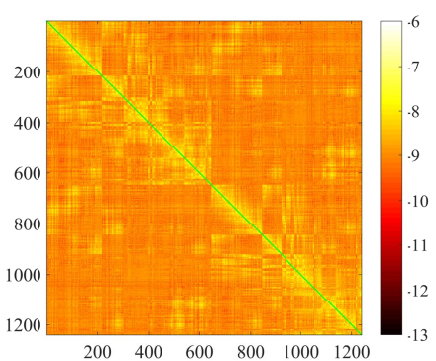

(a)

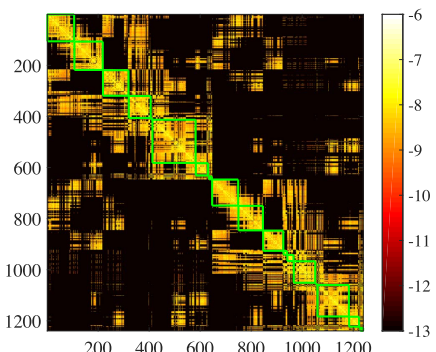

(c)

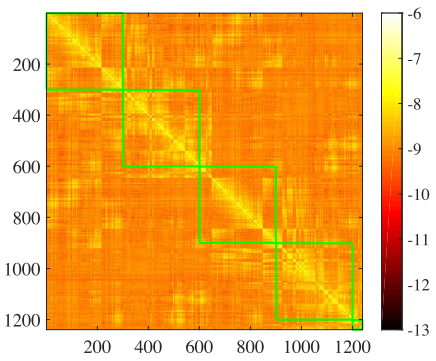

(b)

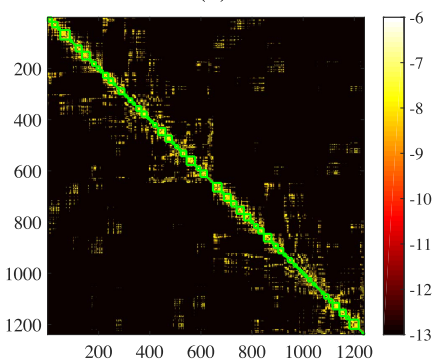

(d)
Fig. 2. (a) Diagonal terms of near-field matrix $\left(\overline{\boldsymbol{\Gamma}}_{J} J\right)$ which are used to construct the left preconditioner at level 2. (b) Block diagonal terms of nearfield matrix $\left(\overline{\boldsymbol{\Gamma}}_{\boldsymbol{J}, \boldsymbol{J}}\right)$ which are used to construct the left preconditioner at level 2, (c) level 3, and (d) level 4 according to (12).

TABLE I

NUMBER OF ITERATIONS BASED ON THE RESIDUAL ERROR $10^{-3}$ FOR THE SOLUTION OF THE PEC SPHERE PROBLEM

\begin{tabular}{|c|c|c|c|c|}
\hline \multicolumn{2}{c}{ Sphere Problem } & \multicolumn{3}{c|}{ Potential Based Integral Equations } \\
\hline \hline Level & Unknowns & NP & D & BD \\
\hline 2 & 2065 & 488 & 27 & 19 \\
\hline 3 & 2065 & 491 & 30 & 18 \\
\hline 4 & 2065 & 492 & 23 & 21 \\
\hline \multicolumn{2}{|c|}{ NP: No preconditioner, D: Diagonal, BD: Block diagonal } \\
\hline
\end{tabular}

one single testing function, the final expression at the end of the QR-decomposition algorithm is obtained as

$$
\begin{aligned}
\sum_{p} J_{p} \frac{\mathrm{e}^{i k R}}{4 \pi R} \simeq & \frac{i k C}{(4 \pi)^{2}} \underbrace{\Psi\left(\hat{\boldsymbol{k}}_{n_{s}}\left(\theta_{n_{s \theta}}, \varphi_{n_{s \varphi}}\right)\right)^{T}}_{\text {Aggregation and disaaggregation }} \\
& \cdot \underbrace{\left(\overline{\mathbf{R}}_{11}^{-1} \cdot \overline{\mathbf{Q}}_{11}^{-1} \cdot \overline{\mathbf{y}}^{T}\right) \cdot \tilde{\boldsymbol{T}}_{Z}\left(k \boldsymbol{w}, \theta_{n_{\theta}}+i \chi, \varphi_{n_{\varphi}}\right)}_{\text {New translation }} \\
& \forall n_{s \theta} \in[1,(L+1)], \forall n_{s \varphi} \in[1,(L+1)]
\end{aligned}
$$

where

$$
\begin{aligned}
{[\overline{\mathbf{y}}]_{\left(N_{\varphi}\left(n_{\theta}-1\right)+n_{\varphi}\right), l(l+1)+m+1=} } & j_{l}\left(2 k r_{C}\right) \\
& \cdot Y_{l, m}\left(\overline{\mathbf{R}}_{v} \cdot \hat{\boldsymbol{k}}_{n}\left(\theta_{n_{\theta}}+i \chi, \varphi_{n_{\varphi}}\right)\right) \\
& \forall l \in[0, L], \forall m \in[-l, l] . \quad(1
\end{aligned}
$$

In (10), $\left[\Psi\left(\hat{\boldsymbol{k}}_{n_{s}}\left(\theta_{n_{s} \theta}, \varphi_{n_{s \varphi}}\right)\right)\right]_{(L+1)^{2} \times 1}$ is a vector containing the new discretized shift functions (i.e., the new discretized points $\theta_{n_{s} \theta}$ and $\varphi_{n_{s \varphi}}$ ) that represent the aggregation and disaggregation stages. In addition, $\overline{\mathbf{R}}_{11}$ and $\overline{\mathbf{Q}}_{11}$ with dimensions of $(L+1)^{2} \times(L+1)^{2}$ are extracted submatrices from upper triangular and unitary matrices, respectively, obtained from QR-decomposition algorithm. In the same equation, $[\overline{\mathbf{y}}]$ contains the spherical harmonics $Y_{l, m}\left(\overline{\mathbf{R}}_{v} \cdot \hat{\boldsymbol{k}}_{n}\right)$ of degree $l$ and order $m$. Spherical Bessel function $j_{l}\left(2 k r_{C}\right)$ is

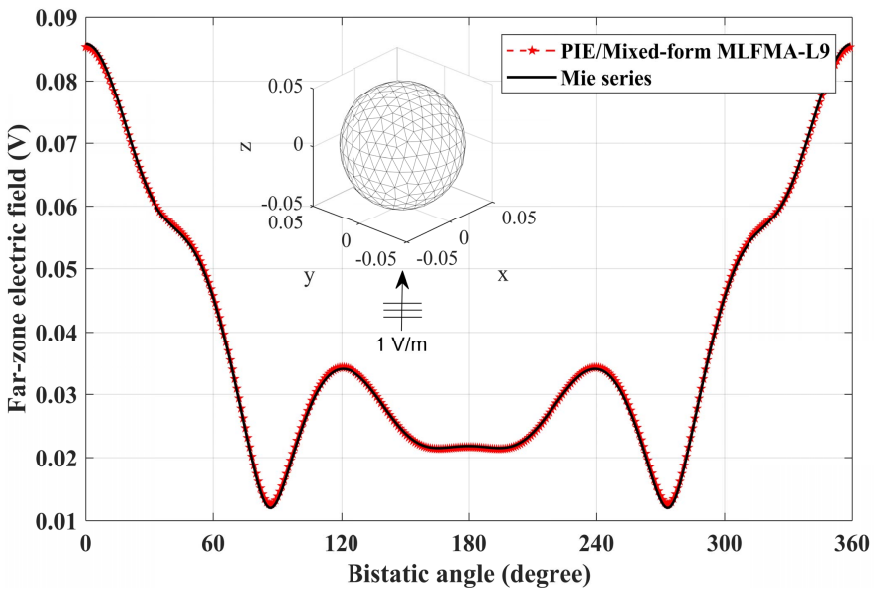

(a)

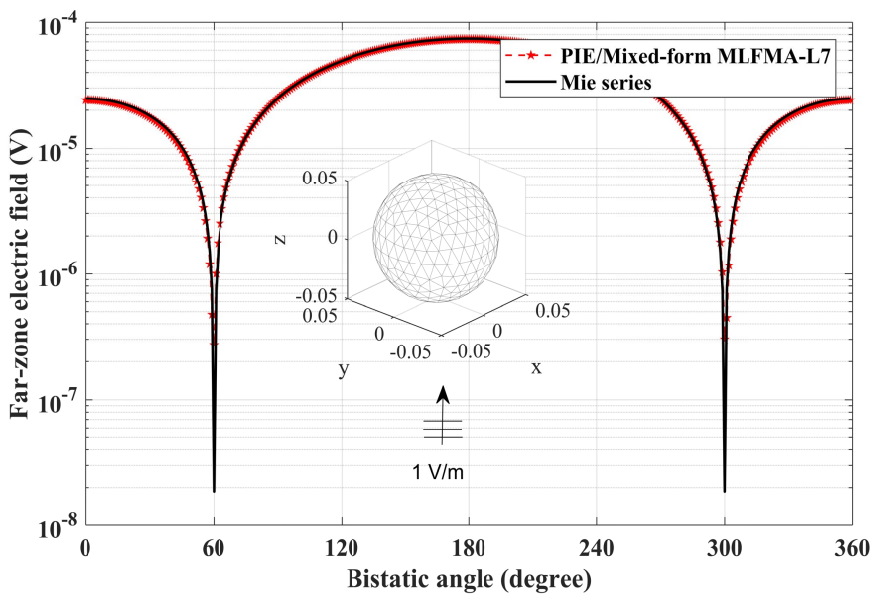

(b)

Fig. 3. Far-zone scattered electric field results obtained from numerical and analytical solutions for a PEC sphere of diameter $10 \mathrm{~cm}$ at (a) $3 \mathrm{GHz}$ and (b) $30 \mathrm{MHz}$.

multiplied as a scaling factor in order to compensate for the grow of spherical function at higher degree, where $r_{C}$ represents the distance from the center of cube to its vertex. Hence, $\left(\overline{\mathbf{R}}_{11}^{-1} \cdot \overline{\mathbf{Q}}_{11}^{-1} \cdot \overline{\mathbf{y}}^{T}\right)$. $\tilde{\boldsymbol{T}}_{Z}\left(k \boldsymbol{w}, \theta_{n_{\theta}}+i \chi, \varphi_{n_{\varphi}}\right)$ represents the new translation in the desired direction.

Implementation of NSPWMLFMA for the solution of PIEs requires the construction of two separate tree structures for the RWG and pulse basis functions. It should be noted that the box indices in tree structures are not identical, which requires to uniquely define the translations among the boxes containing RWGs and the boxes containing pulses. In other words, in addition to translations among the boxes containing RWG basis functions (i.e., RWG to RWG), new translations among the boxes containing pulses (i.e., pulse to pulse), and from the boxes containing RWG basis functions to boxes containing pulse basis functions (i.e., RWG to pulse) and vice versa (i.e., pulse to RWG) are uniquely defined, thereby assuring the desired accuracy.

\section{Preconditioning for PIE and Mixed-Form MLFMA}

In a similar fashion to [16], where a comprehensive study for conditioning properties of PIEs has been provided, a left constraint preconditioner $\overline{\mathbf{P}}_{C}^{-1}$ is used for $\overline{\mathbf{Z}}=\left[\begin{array}{cc}\overline{\boldsymbol{\Gamma}}_{\boldsymbol{J}} \boldsymbol{J} & \overline{\boldsymbol{\Gamma}}_{\boldsymbol{J}}, \sigma \\ \overline{\boldsymbol{\Gamma}}_{\sigma, \boldsymbol{J}} & k_{0}^{2} \overline{\boldsymbol{\Gamma}}_{\sigma, \sigma}\end{array}\right]$ to reduce the number of iterations. 


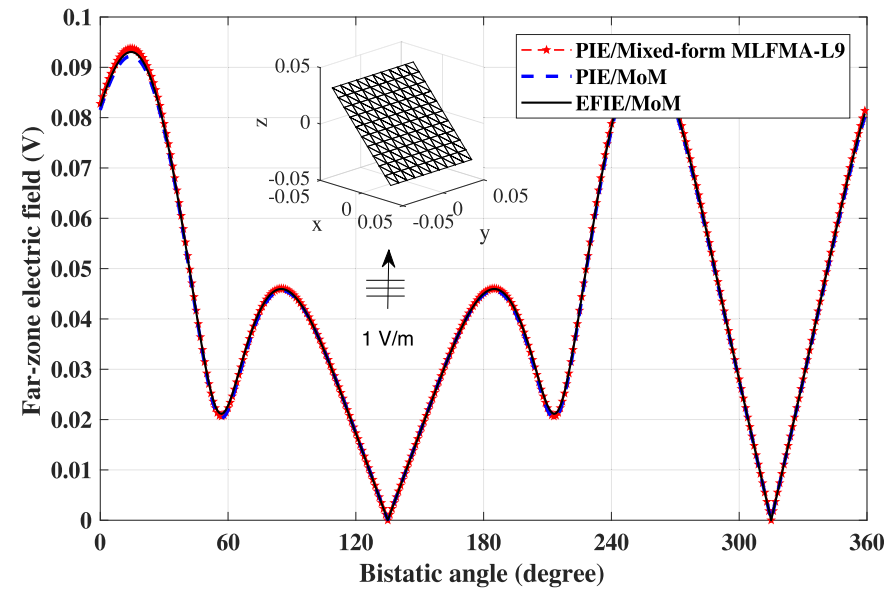

(a)

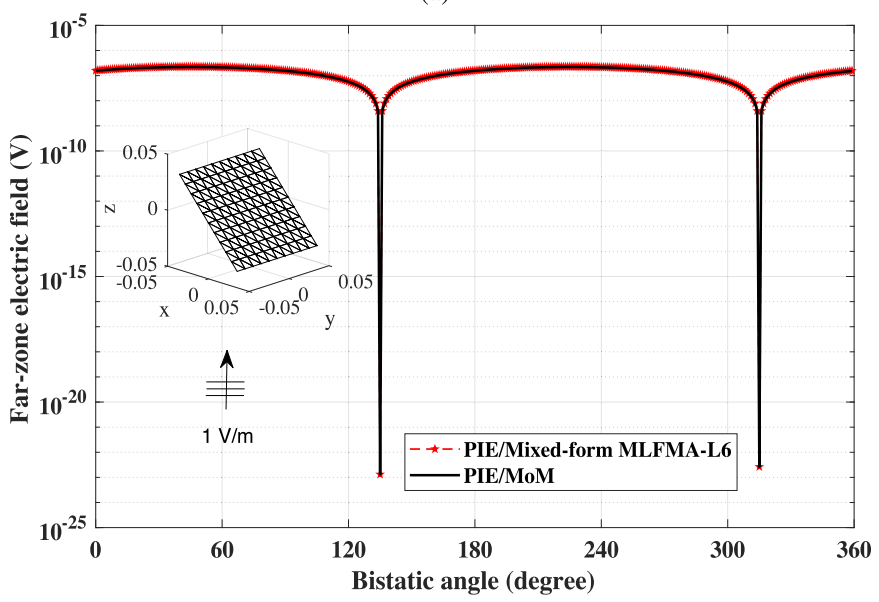

(b)

Fig. 4. Far-zone scattered electric field results obtained from numerical solutions for a PEC plate with edge size of $10 \mathrm{~cm}$ discretized with different mesh sizes including fine and coarse cases at (a) $3 \mathrm{GHz}$ and (b) $3 \mathrm{MHz}$.

Briefly, $\overline{\mathbf{P}}_{C}^{-1}$ is obtained as

$$
\overline{\mathbf{P}}_{C}^{-1}=\left[\begin{array}{cc}
\overline{\mathbf{I}} & \overline{\mathbf{D}}^{-1} \overline{\mathbf{\Gamma}}_{J, \sigma} \\
\overline{\mathbf{0}} & \overline{\mathbf{I}}
\end{array}\right]\left[\begin{array}{cc}
\overline{\mathbf{D}}^{-1} & \overline{\mathbf{0}} \\
\overline{\mathbf{0}} & \overline{\mathbf{S}}^{-1}
\end{array}\right]\left[\begin{array}{cc}
\overline{\mathbf{I}} & \overline{\mathbf{0}} \\
-\overline{\mathbf{\Gamma}}_{\sigma, J} \overline{\mathbf{D}}^{-1} & \overline{\mathbf{I}}
\end{array}\right]
$$

where $\overline{\mathbf{S}}=-\left(-k_{0}^{2} \overline{\boldsymbol{\Gamma}}_{\sigma, \sigma}+\overline{\boldsymbol{\Gamma}}_{\sigma, \boldsymbol{J}}(\overline{\mathbf{D}})^{-1} \overline{\boldsymbol{\Gamma}}_{\boldsymbol{J}, \sigma}\right)$ is the Schur complement of $\overline{\mathbf{P}}_{C}$, and $\mathbf{D}$ is the diagonal form of $\overline{\boldsymbol{\Gamma}}_{\boldsymbol{J}, \boldsymbol{J}}$ as an approximation. Note that instead of $\overline{\mathbf{D}}$, one can use $\overline{\mathbf{D}}^{\prime}$ formed from the block diagonal elements of $\overline{\boldsymbol{\Gamma}}_{\boldsymbol{J}, \boldsymbol{J}}$ that are obtained from near-field interactions as explained in [33]. This can reduce the number of iterations even further. Other matrices will remain intact, which are obtained from their corresponding near-field interactions. For a PEC sphere discretized with 826 triangles (total number of unknowns $N+M=2065$ ), Fig. 2 shows both the diagonal and block diagonal terms of $\overline{\boldsymbol{\Gamma}}_{\boldsymbol{J}, \boldsymbol{J}}$ used in $\overline{\mathbf{P}}_{C}^{-1}$ at different mixed-form MLFMA levels, where the generalized minimal residual (GMRES) method iterative solver with an error tolerance of $10^{-3}$ is used with maximum number of iterations fixed to 1000 . Table I shows the number of iterations when there is no preconditioner, and when $\overline{\mathbf{D}}$ and $\overline{\mathbf{D}}^{\prime}$ as a diagonal and block diagonal approximations are used in (12), respectively. Here, level 2 corresponds to the MoM solution. It can be seen that the use of $\overline{\mathbf{D}}^{\prime}$ reduces the total iteration counts to reasonable numbers.

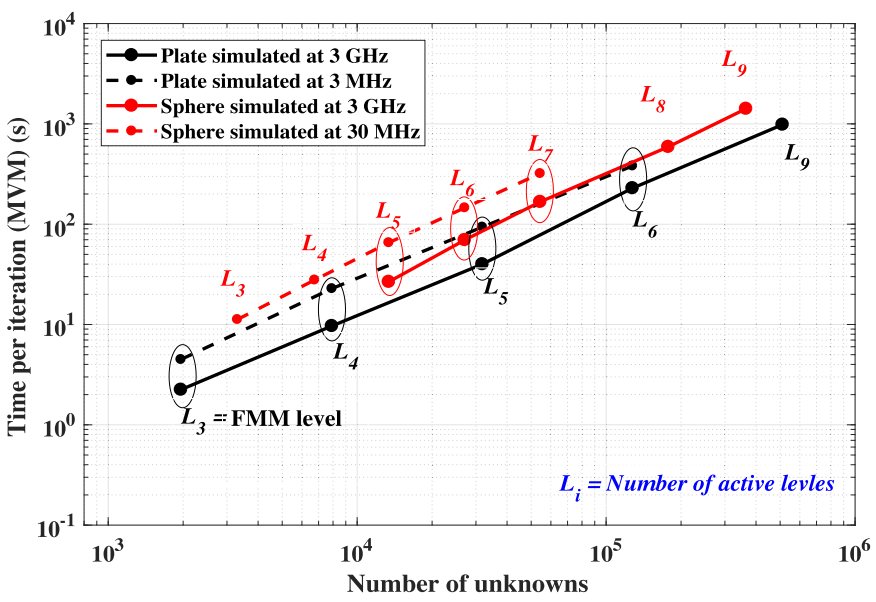

Fig. 5. Matrix-vector multiplication (MVM) times for both a PEC tilted plate and a PEC sphere at two different frequencies (3 GHz and $3 \mathrm{MHz}$ for tilted PEC plate, and $3 \mathrm{GHz}$ and $30 \mathrm{MHz}$ for the PEC sphere). For the PEC tilted plate, the number of unknowns is increasing from 1960 to 511360 by refining the discretization from $\lambda / 20$ to $\lambda / 320$ at $3 \mathrm{GHz}$ and from 1960 to 127680 by refining the discretization from $\lambda / 20000$ to $\lambda / 160000$ at $3 \mathrm{MHz}$. Minimum box sizes in the tree structures for $3 \mathrm{GHz}$ and $3 \mathrm{MHz}$ are $\lambda / 256$ and $\lambda / 32000$, respectively. For the PEC sphere, these numbers as follows: the number of unknowns is increasing by refining the discretizations from 13405 $\left(l_{\lambda}=\lambda / 30\right)$ to $364865\left(l_{\lambda}=\lambda / 150\right)$ at $3 \mathrm{GHz}$, and from $3305\left(l_{\lambda}=\lambda / 1400\right)$ to $54365\left(l_{\lambda}=\lambda / 5600\right)$ at $30 \mathrm{MHz}$. Minimum box sizes in the tree structures for $3 \mathrm{GHz}$ and $30 \mathrm{MHz}$ are $\lambda / 256$ and $\lambda / 6400$, respectively.

\section{NUMERICAL RESULTS}

Numerical results are presented to illustrate the capability of the proposed mixed-form MLFMA solver for PIEs, where GMRES method with an error tolerance of $10^{-4}$ is used as the iterative solver. Number of harmonics, $L$, at low frequencies (i.e., electrically small boxes) is fixed (regardless of the box size) [31], [32] and is selected to be 25 for all the numerical examples presented in this communication. At middle and high frequencies, where the box sizes are comparable to the wavelength, excess bandwidth formula is used [2]. In the simulations, $2 L+2$ integration points are used both for $\theta$ and $\varphi$ directions. All of the results are obtained by a doubleprecision program on a cluster of $\operatorname{Intel}(\mathrm{R}) \mathrm{Xeon}(\mathrm{R}) \mathrm{X} 5472$ processors with $3.00 \mathrm{GHz}$ clock rate and $32 \mathrm{~GB}$ RAM.

The first example is a PEC sphere with a diameter of $0.1 \mathrm{~m}$, which is illuminated by an $x$-polarized uniform plane-wave propagating along the $z$-direction. Fig. 3(a) shows the far-zone scattered electric field as a function of the bistatic angle on the $z x$ plane $\left(0^{\circ}\right.$ and $180^{\circ}$ represent forward and backward scattering directions, respectively) compared with the Mie series result when the operating frequency is $3 \mathrm{GHz}$. The far-zone electric field is defined as the electric-field intensity multiplied by the distance from the object when the distance goes to infinity. At this frequency, the diameter of the sphere, $d_{\lambda}$, corresponds to $\lambda$ and each triangle has an approximate edge length, $l_{\lambda}$, of $\lambda / 150$. A total of 364865 unknowns are solved using ninelevel mixed-form MLFMA. Fig. 3(b) shows a similar result when the frequency is $30 \mathrm{MHz}$, where $d_{\lambda}=\lambda / 100$ and $l_{\lambda}=\lambda / 5600$. In this case, 54365 unknowns are solved with a seven-level mixedform MLFMA. The number of iterations is 86 and 59 at $3 \mathrm{GHz}$ and $30 \mathrm{MHz}$, respectively. In both cases, a very good agreement is obtained, which verifies the accuracy of the proposed method at both high and low frequencies.

A tilted $10 \mathrm{~cm} \times 10 \mathrm{~cm}$ PEC plate, illuminated by an $x$-polarized uniform plane-wave propagating along the $z$-direction, is the second example. Similar to the PEC sphere case, two different operating frequencies are selected as $3 \mathrm{GHz}$ and $3 \mathrm{MHz}$. At $3 \mathrm{GHz}$, the plate is 


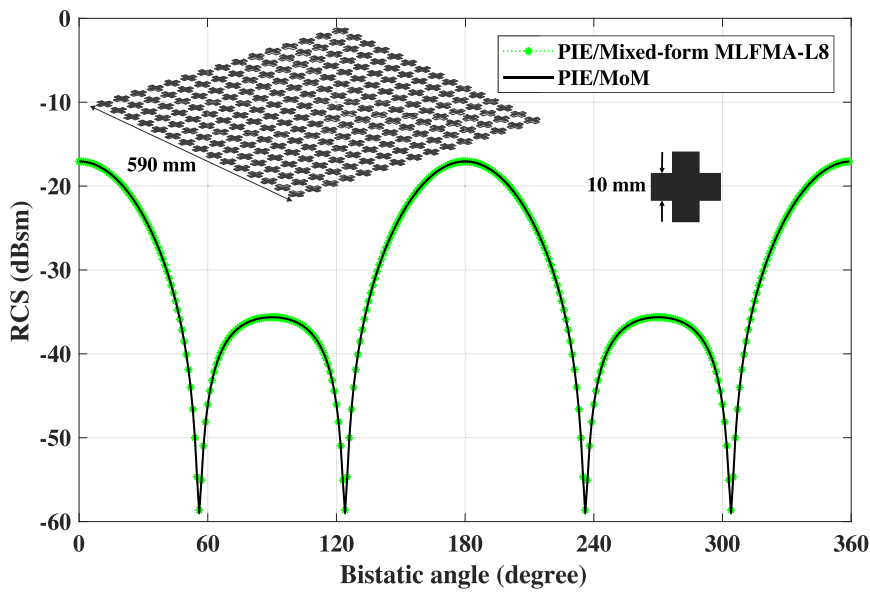

Fig. 6. RCS results obtained by using two different mesh sizes $l_{\lambda}=\lambda / 100$ and $l_{\lambda}=\lambda / 200$, which are corresponding to 23175 and 119175 unknowns at $600 \mathrm{MHz}$. The coarse and fine discretized objects are solved by using MoM and 8-level mixed-form MLFMA with approximate minimum box sizes of $\lambda / 128$ in the tree structure.

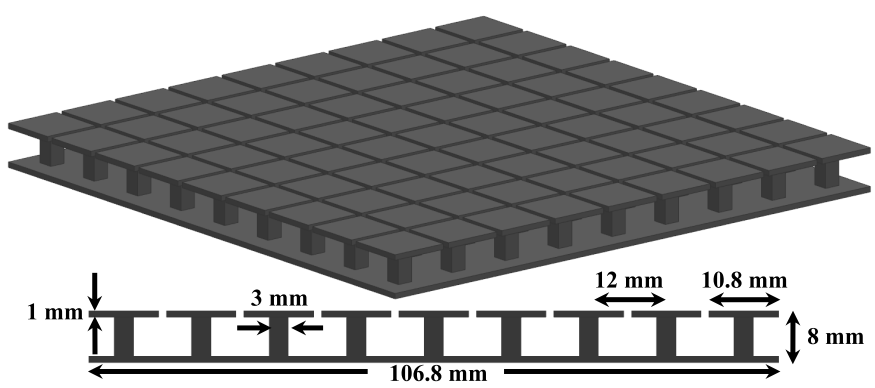

Fig. 7. Geometry of a mushroom-type structure.

$\lambda \times \lambda$ and discretized with triangles of edge size $l_{\lambda}=\lambda / 320$. A total of 511360 unknowns are solved with a 9-level mixed-form MLFMA in 79 iterations. The scattered far-field result is compared with both PIEs and EFIE solved with MoM by using coarse discretization $\left(l_{\lambda}=\lambda / 10\right)$ as shown in Fig. 4(a), where an excellent agreement can be seen. The same plate is $\lambda / 1000 \times \lambda / 1000$ at $3 \mathrm{MHz}$ and discretized with triangles of edge size $l_{\lambda}=\lambda / 160000$ leading to 127680 unknowns, which are solved with a 6-level mixed-form MLFMA. For the solution, 55 iterations are required to reach $10^{-4}$ residual error. The far-field result is compared with PIE/MoM in Fig. 4(b), where an excellent agreement is obtained.

Fig. 5 presents the running time per iteration versus the number of unknowns both for the PEC sphere and the tilted PEC plate examples at two different frequencies $(3 \mathrm{GHz}$ and $30 \mathrm{MHz}$ for the PEC sphere and $3 \mathrm{GHz}$ and $3 \mathrm{MHz}$ for tilted PEC plate). An $O(N L)$ complexity, where $L$ is the number of levels, can be observed for the CPU time.

The third example is a 2-D periodic electromagnetic band gap structure formed of PEC cross-shaped elements as shown in Fig. 6. The PEC structure is illuminated by a uniform plane-wave polarized in both $x$ - and $y$-directions and propagating along the $-z$ direction [i.e., $\boldsymbol{E}=(1 / \sqrt{2})\left(\hat{\boldsymbol{a}}_{x}+\hat{\boldsymbol{a}}_{y}\right)$ and $\hat{\boldsymbol{k}}=-\hat{\boldsymbol{a}}_{z}$ ] at $600 \mathrm{MHz}$. Radar cross section (RCS) is obtained both with MoM (used to solve PIEs) and eight-level mixed-form MLFMA. Excellent agreement is obtained as can be seen from Fig. 6. A total of 119175 unknowns are used in this example for the mixed-form MLFMA solver. Note that, because of memory and CPU time restrictions, a coarser mesh with 23175 unknowns is used for the MoM solution.

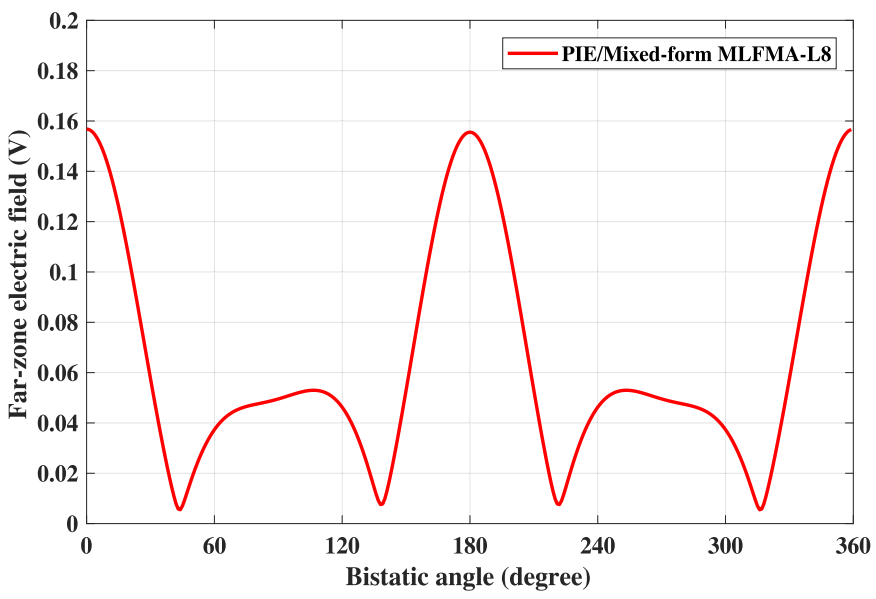

Fig. 8. Far-zone scattered electric field result obtained from eight-level mixed-form MLFMA solution of the PEC mushroom-type structure at $3 \mathrm{GHz}$.

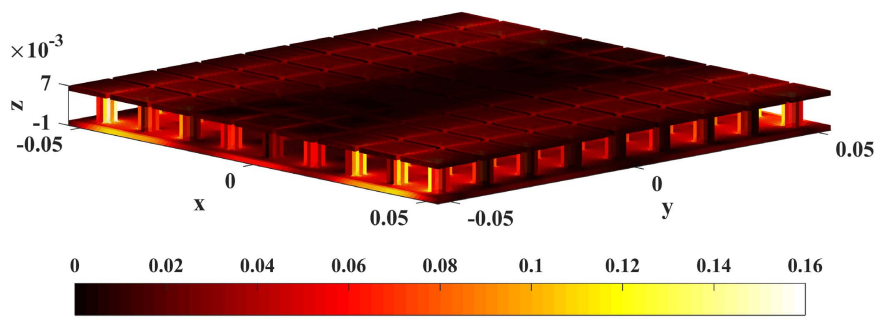

Fig. 9. Linear current distribution of the mushroom-type structure illuminated by a uniform plane-wave polarized in $x$-direction.

Final example is grounded mushroom-type structure with total dimensions of $10.68 \mathrm{~cm} \times 10.68 \mathrm{~cm} \times 0.8 \mathrm{~cm}$ as shown in Fig. 7 [34]. The structure is discretized with a total of 394710 unknowns, corresponding to $1 \mathrm{~mm}$ triangle edge, and solved with eight-level mixed-form MLFMA at $3 \mathrm{GHz}$ for an $x$-polarized plane-wave propagating along the $-z$ direction. The far-zone scattered electric field and linear current distribution results are presented in Figs. 8 and 9, respectively.

\section{CONCLUSION}

In this contribution, we propose a method to solve densely discretized objects over a wide frequency range including low frequencies by combining a mixed-form MLFMA with the recently introduced PIEs. The mixed-form MLFMA uses NSPWMLFMA at lower frequencies (i.e., at the deeper levels in the tree structures when the box sizes are less than $\lambda / 8$ ) and the conventional MLFMA for middle/higher frequencies. Consequently, it does not suffer from LF breakdown of the conventional MLFMA and SIEs. It is also applicable to both open surfaces and closed objects. Various geometries are investigated in terms of scattered fields and current distribution and compared with analytical and MoM solutions (the latter obtained with coarse meshes due to complexity issues) that verify the accuracy and efficiency of the method.

\section{REFERENCES}

[1] R. F. Harrington, Field Computation by Moment Methods. New York, NY, USA: Macmillan, 1993.

[2] W. C. Chew, E. Michielssen, J. M. Song, and J. M. Jin, Fast and Efficient Algorithm in Computational Electromagnetics. Norwood, MA, USA: Artech House, 2001. 
[3] Ö. Ergül and L. Gürel, The Multilevel Fast Multipole Algorithm (MLFMA) for Solving Large-Scale Computational Electromagnetic Problems. Piscataway, NJ, USA: IEEE Press, 2014.

[4] J. Zhu, S. Omar, and D. Jiao, "Solution of the electric field integral equation when it breaks down," IEEE Trans. Antennas Propag., vol. 62, no. 8, pp. 4122-4134, Aug. 2014.

[5] K. Cools, F. P. Andriulli, D. D. Zutter, and E. Michielssen, "Accurate and conforming mixed discretization of the MFIE," IEEE Antennas Wireless Propag. Lett., vol. 10, pp. 528-531, May 2011.

[6] Ö. Ergül and L. Gürel, "Improved testing of the magnetic-field integral equation," IEEE Microw. Wireless Compon. Lett., vol. 15, no. 10, pp. 615-617, Oct. 2005

[7] G. Vecchi, "Loop-star decomposition of basis functions in the discretization of the EFIE," IEEE Trans. Antennas Propag., vol. 47, no. 2 , pp. 339-346, Feb. 1999.

[8] M. Taskinen and P. Yla-Oijala, "Current and charge integral equation formulation," IEEE Trans. Antennas Propag., vol. 54, no. 1, pp. 58-67, Jan. 2006.

[9] Z.-G. Qian and W. C. Chew, "Enhanced A-EFIE with perturbation method," IEEE Trans. Antennas Propag., vol. 58, no. 10, pp. 3256-3264, Oct. 2010.

[10] K. Cools, F. P. Andriulli, F. Olyslager, and E. Michielssen, "Nullspaces of MFIE and calderón preconditioned EFIE operators applied to toroidal surfaces," IEEE Trans. Antennas Propag., vol. 57, no. 10, pp. 3205-3215, Oct. 2009.

[11] F. P. Andriulli, K. Cools, I. Bogaert, and E. Michielssen, "On a well-conditioned electric field integral operator for multiply connected geometries," IEEE Trans. Antennas Propag., vol. 61, no. 4 pp. 2077-2087, Apr. 2013.

[12] F. P. Andriulli, F. Vipiana, and G. Vecchi, "Hierarchical bases for nonhierarchic 3-D triangular meshes," IEEE Trans. Antennas Propag., vol. 56, no. 8, pp. 2288-2297, Aug. 2008.

[13] M. A. E. Bautista, M. A. Francavilla, F. Vipiana, and G. Vecchi, "A hierarchical fast solver for EFIE-MoM analysis of multiscale structures at very low frequencies," IEEE Trans. Antennas Propag., vol. 62, no. 3, pp. 1523-1528, Mar. 2014.

[14] F. Vico, M. Ferrando, L. Greengard, and Z. Gimbutas, "The decoupled potential integral equation for time-harmonic electromagnetic scattering," Commun. Pure Appl. Math., vol. 69, no. 4, pp. 771-812, Apr. 2016.

[15] W. C. Chew, "Vector potential electromagnetics with generalized gauge for inhomogeneous media: Formulation," Prog. Electromagn. Res., vol. 149, pp. 69-84, Jan. 2014.

[16] Q. S. Liu, S. Sun, and W. C. Chew, "A potential-based integral equation method for low-frequency electromagnetic problem," IEEE Trans. Antennas Propag., vol. 66, no. 3, pp. 1413-1426, Mar. 2018.

[17] U. M. Gür and Ö. Ergül, "Accuracy of sources and near-zone fields when using potential integral equations at low frequencies," IEEE Antennas Wireless Propag. Lett., vol. 16, pp. 2783-2786, Aug. 2017.

[18] H. Wallén and J. Sarvas, "Translation procedures for broadband MLFMA," Prog. Electromagn. Res., vol. 55, pp. 47-78, Jan. 2005.

[19] I. Bogaert and F. Olyslager, "A low-frequency stable plane wave addition theorem," J. Comput. Phys., vol. 228, no. 4, pp. 1000-1016, Mar. 2009.
[20] I. Bogaert, J. Peeters, and F. Olyslager, "A nondirective plane wave MLFMA stable at low frequencies," IEEE Trans. Antennas Propag., vol. 56, no. 12, pp. 3752-3767, Dec. 2008.

[21] J. Peeters, K. Cools, I. Bogaert, F. Olyslager, and D. D. Zutter, "Embedding Calderon multiplicative preconditioners in multilevel fast multipole algorithms," IEEE Trans. Antennas Propag., vol. 58, no. 4, pp. 1236-1250, Apr. 2010.

[22] L. Greengard, J. Huang, V. Rokhlin, and S. Wandzura, "Accelerating fast multipole methods for the Helmholtz equation at low frequencies," IEEE Comput. Sci. Eng., vol. 5, no. 3, pp. 32-38, Jul./Sep. 1998.

[23] L. J. Jiang and W. C. Chew, "Low-frequency fast inhomogeneous plane-wave algorithm," Microw. Opt. Technol. Lett., vol. 40, no. 2, pp. 117-122, Jan. 2004

[24] E. Darve and P. Have, "Efficient fast multipole method for low-frequency scattering," J. Comput. Phys., vol. 197, no. 1, pp. 341-363, Jun. 2004.

[25] H. Cheng et al, "A wideband fast multipole method for Helmholtz equation in three dimensions," J. Comput. Phys., vol. 216, no. 1, pp. 300-325, Jul. 2006.

[26] M. Vikram, H. Huang, B. Shanker, and T. Van, "A novel wideband FMM for fast integral equation solution of multiscale problems in electromagnetics," IEEE Trans. Antennas Propag., vol. 57, no. 7, pp. 2094-2104, Jul. 2009.

[27] Ö. Ergül and B. Karaosmanoglu, "Broadband multilevel fast multipole algorithm based on an approximate diagonalization of the Green's function," IEEE Trans. Antennas Propag., vol. 63, no. 7, pp. 3035-3041, Jul. 2015.

[28] M. Takrimi, Ö. Ergü, and V. B. Ertürk, "A novel broadband multilevel fast multipole algorithm with incomplete-leaf tree structures for multiscale electromagnetic problems," IEEE Trans. Antennas Propag., vol. 64 , no. 6, pp. 2445-2456, Jun. 2016.

[29] D. T. Schobert and T. F. Eibert, "Low-frequency surface integral equation solution by multilevel Green's function interpolation with fast Fourier transform acceleration," IEEE Trans. Antennas Propag., vol. 60, no. 3 , pp. 1440-1449, Mar. 2012.

[30] S. Rao, D. Wilton, and A. Glisson, "Electromagnetic scattering by surfaces of arbitrary shape," IEEE Trans. Antennas Propag., vol. AP-30, no. 3, pp. 409-418, May 1982.

[31] I. Bogaert, J. Peeters, and D. D. Zutter, "Error control of the vectorial nondirective stable plane wave multilevel fast multipole algorithm," Prog. Electromagn. Res., vol. 111, pp. 271-290, Jan. 2011.

[32] M. Kalfa, Ö. Ergül, and V. B. Ertürk, "Error control of multipleprecision MLFMA," IEEE Trans. Antennas Propag., vol. 66, no. 10, pp. 5651-5656, Oct. 2018.

[33] T. Malas and L. Gürel, "Schur complement preconditioners for surface integral-equation formulations of dielectric problems solved with the multilevel fast multipole algorithm," SIAM J. Sci. Comput., vol. 33, no. 5 , pp. 2440-2467, 2011.

[34] O. Luukkonen, M. G. Silveirinha, A. B. Yakovle, C. R. Simovski, I. S. Nefedov, and S. A. Tretyakov, "Effects of spatial dispersion on reflection from mushroom-type artificial impedance surfaces," IEEE Trans. Microw. Theory Techn., vol. 57, no. 11, pp. 2692-2699, Nov. 2009. 